\title{
HORMIGONES DE ULTRA ALTO DESEMPEÑO: DISEÑO PARA UNA ALTA RESISTENCIA A LA COMPRESIÓN (138 MEGAPASCAL) Y A LA EROSIÓN-ABRASIÓN MANTENIENDO ALTA TRABAJABILIDAD
}

\author{
ULTRA HIGH PERFORMANCE CONCRETE: DESIGN FOR HIGH \\ COMPRESSIVE STRENGTH (138 MEGAPASCAL) AND ABRASION \\ WHILE MAINTAINING HIGH WORKABILITY
}

FABRICIO YÉPEZ

Universidad de San Francisco de Quito. Ecuador. fyepez@usfq.edu.ec

RESUMEN

El desarrollo y producción de hormigones de alto desempeño o HPC (High Performance Concrete) han evidenciado un avance lento en Ecuador. En este artículo se reportan los resultados de una investigación que tuvo como objetivo la obtención de una dosificación volumétrica de hormigón capaz de cumplir tres requerimientos de desempeño: proporcionar una resistencia a la compresión mayor a los 90 megapascal, en conjunto con una adecuada trabajabilidad de mezcla y, a su vez, presentar una muy alta resistencia a la erosión abrasión, requerimientos necesarios para el revestimiento de los desagües de fondo de algunos proyectos hidroeléctricos en Ecuador. Asimismo, este trabajo pretende servir como quna guía y punto de partida para el desarrollo de hormigones de alto y ultra alto desempeño. La investigación toma en cuenta consideraciones económicas, así como disponibilidad de los materiales en el mercado local y la relación resistencia/costo del producto final. Para estos fines se desarrollaron varios diseños distintos de mezclas utilizando microsílice ASTM C1240 y un aditivo reductor de agua de alto rango de última generación acorde con ASTM C494. Como resultado final, se obtuvo una mezcla de alta trabajabilidad con una resistencia última a la compresión de $98 \mathrm{MPa}$ a una edad temprana de siete días, con resistencia máxima $138 \mathrm{MPa}$ a los 28 días, siendo hasta hoy, el hormigón de mayor resistencia producido en Ecuador, y con porcentajes de desgaste en relación al peso según ensayo ASTM C1138 que no superan el $0.8 \%$.

PALABRAS CLAVE. hormigón ultra alto desempeño, microsílice, resistencia a la erosión abrasión.
ABSTRACT Performance Concrete) have evidenced a slow progress in Ecuador. This article reports the results of a research that aimed to obtain a volumetric dosing of concrete capable of meeting three performance requirements: to provide a compressive strength greater than 90 megapascal, in conjunction with a suitable mixability and, in turn, present a very high resistance to abrasion erosion, necessary requirements for the lining of the bottom drains of some hydroelectric projects in Ecuador. Also, this work aims to serve as a guide and starting point for the development of high and ultra high performance concrete. The research takes into account economic considerations, as well as availability of the materials in the local market and the resistance / cost ratio of the final product. For these purposes, several different blend designs were developed using ASTM C1240 microsilica and a high-end state-of-the-art water-reducing additive conforming to ASTM C494. As a final result, a high workability blend was obtained with a final compressive strength of $98 \mathrm{MPa}$ at an early age of seven days, with a maximum strength of $138 \mathrm{MPa}$ at 28 days, and to date, the highest strength concrete produced in Ecuador, and with percentages of wear in relation to the weight according to test ASTM C1138 that do not surpass $0.8 \%$.

KEYWORDS: Ultra high performance concrete, microsilice, erosion resistance abrasion. 
INTRODUCCIÓN

Siendo el hormigón uno de los materiales más utilizados a nivel mundial y, observando las limitaciones de esta industria en Ecuador, surge la necesidad de desarrollar un método coherente y seguro que lleve al desarrollo consistente de mezclas de hormigones de alto desempeño, esto es, que diferentes requerimientos de un proyecto puedan ser satisfechos por un hormigón de características especiales, y que adicionalmente presente una alta resistencia a la compresión. Por otro lado, la ejecución de diversos proyectos hidroeléctricos en el país requiere la utilización de un material altamente resistente a la erosión abrasión, a ser utilizado como recubrimiento de los desagües de fondo de las presas y aliviaderos. En tal sentido, se requiere diseñar un hormigón de alto desempeño, que presente alta resistencia a la compresión, buena trabajabilidad de la mezcla y alta resistencia a la erosión - abrasión, a través de una metodología consistente para la obtención de una dosificación volumétrica mediante el uso de materiales y métodos disponibles en Ecuador.

El desarrollo del diseño de mezcla utilizará humo de sílice ASTM C1240 y un aditivo reductor de agua de alto rango de última generación, acorde a ASTM C494, cuidando de cerca la relación agua/cementante de la mezcla debido a los buenos resultados de estas prácticas reportados alrededor del mundo. Para desarrollar la mezcla se ha utilizado como base la guía ACI 211.4R-08 [1] y las recomendaciones de Willie, K. et al. [2]. Se discuten luego los procedimientos seguidos para el desarrollo de varias mezclas distintas, los resultados obtenidos, y los costos estimados para una producción industrial acorde a precios del mercado ecuatoriano.

\section{MATERIALES Y METODOLOGÍA} MATERIALES

Cada una de las mezclas preparadas utilizó como materiales una combinación específica de un tipo de cemento, agregados finos y gruesos, microsílice, agua, y un aditivo reductor de agua de alto rango. Estas variaciones se requieren debido a que cambios en las composiciones químicas y propiedades físicas de los materiales impactan las $\mathrm{ca}^{-}$ racterísticas finales del hormigón como su resistencia última, permeabilidad, trabajabilidad, resistencia a la erosión abrasión, durabilidad, etc. [1].
CEMENTO

El cemento utilizado es uno de los factores más influyentes en el desarrollo de hormigones de alto desempeño [1]. En la investigación se ha prestado especial atención al efecto que los distintos tipos de cemento pueden tener en la demanda de agua de la mezcla, el comportamiento del aditivo reductor de agua de alto rango y la microsílice, y la ganancia de resistencia del hormigón en función del tiempo.

Es conocido que una presencia mayor al $8 \%$ de $\mathrm{C} 3 \mathrm{~A}$ en la composición química del cemento no es recomendable para el desarrollo de hormigones de alta resistencia, pues la rápida reacción de este compuesto durante el proceso de hidratación produce un aumento del área superficial de las partículas, aumentando la demanda de agua necesaria para rodearlas y produciéndose un aumento en la viscosidad de la mezcla. Los resultados de la investigación de Willie, K. et al. [2] muestran que cementos con un contenido bajo de C3A facilitan la obtención de relaciones agua/cementante bajas y llevan a alcanzar resistencias finales mayores.

TABLA 1. COMPOSICIÓN QUÍMICA DEL CEMENTO CP-II, CONTENIDO DE C3A LIMITADO AL 7.2\%

\begin{tabular}{lr}
\hline COMPosiclón aúímiCA & $\%$ \\
\hline Si02 & 21.9 \\
\hline Al203 & 5.1 \\
\hline Fe203 & 3.7 \\
\hline CaO & 64.4 \\
\hline MgO & 0.8 \\
\hline S03 & 1.8 \\
\hline Na2O & 0.23 \\
\hline K20 & 0.19 \\
\hline V & 0.36 \\
\hline Total & 100 \\
\hline Cal Libre & 0.75 \\
\hline Residuo Insoluble & 0.7 \\
\hline Álcalis Equivalente (\%) & 0.36 \\
\hline POTENciaL & 7.2 \\
\hline C3A & 79.3
\end{tabular}

Otro punto al que se ha prestado atención, es el hecho de que uno de los mecanismos por los cuales la microsílice aporta al aumento de resistencia en la mezcla es reaccionando con hidróxido de calcio $\left(\mathrm{Ca}(\mathrm{OH})_{2}\right.$ denotado también como $\mathrm{CH}$ ) para producir silicato cálcico hidratado (CSH) y otros cementantes adicionales [3]. De esta información se concluye que la com- 
posición química del cemento, que delimita los productos de su hidratación, va a afectar la acción de la microsílice en la mezcla. Con estas ideas en mente, se ha optado por utilizar, a manera de comparación, dos tipos de cementos distintos disponibles en Ecuador: el primero es un cemento portland tipo II (CP-II), de bajo contenido de C3A (tabla 1) y el segundo, un cemento hidráulico que cumple la norma ASTM C1157 de desempeño, para el cual no se dispone de su composición química pero tiene una descripción de alta resistencia temprana según su fabricante (CH-HE).

MICROSÍLICE

La microsílice es definida como un puzolánico producto de desecho de la industria de metales silicoferrosos, utilizada en el desarrollo de hormigones principalmente para mejorar sus propiedades mecánicas y durabilidad. Pese a que actualmente se cuenta con especificaciones suficientes para garantizar su utilización adecuada, se debe tener en cuenta la variabilidad en sus propiedades que, entre otras cosas, depende del tipo de aleación que se esté produciendo en su origen. A manera de ejemplo, se presentan las tablas 2 y 3 en las cuales se muestra un estudio de la variación en la composición química y propiedades físicas de microsílice obtenida de diversas fuentes en Noruega y Norteamérica [3]. Este estudio no ha podido establecer una relación clara entre varios de los parámetros comparados en las tablas y el desempeño de la microsílice en mezclas de hormigón de alto desempeño, ni tampoco una relación importante entre los tipos de microsílice mencionados y la resistencia final del hormigón [3]. Por este motivo, en la presente investigación se ha decidido realizar todas las mezclas con un único tipo de microsílice con el fin de no introducir una cantidad excesiva de variables que pueden escapar al control del productor y tener un impacto desconocido. Se utilizó microsílice de procedencia China, que posee un contenido del $95 \%$ de $\mathrm{SiO}_{2}$ y que cumple con la norma ASTM C1240.

ADITIVO REDUCTOR DE AGUA DE ALTO RANGO (HRWR)

Para hormigones de alta resistencia, uno de los parámetros más críticos es la relación w/c (agua/cementante) [1, 2]. La microsílice es un cementante adicional que, mediante mecanismos físicos y químicos, es capaz de aumentar la resistencia a la compresión del hormigón, pero produce efectos en el hormigón fresco que deben ser tomados en cuenta [1, 3]. Ésta produce un aumento en la demanda de agua debido a su alta área superficial, además de proporcionar viscosidad al hormigón, lo cual disminuye su trabajabilidad [1, 3, 4]. Por ello, el desarrollo de hormigones de alto desempeño necesita de la incorporación de un HRWR (high range water reducer). Estos aditivos son capaces de reducir el agua de la mezcla incluso en un porcentaje superior al 30\% [5]; de otra manera, el agua adicional necesaria para proporcionar al hormigón la trabajabilidad adecuada, acarrearía un aumento en la relación w/c y con esto una disminución de la resistencia $[1,3]$.

TABLA 2. VARIACIONES EN LA COMPOSICIÓN QUÍMICA DE MICROSÍLICE RECOPILADAS DE DISTINTAS FUENTES POR ACI-211

\begin{tabular}{|c|c|c|c|c|c|c|c|c|c|c|}
\hline TIPO DE ALEACIÓN & & SI & & FESI-75\% & & Y FESI-75\% & & FESI-75\% & & sI \\
\hline \multirow[t]{2}{*}{ NUMERO DE MUESTRAS } & & 42 & & 42 & & 32 & & 6 & & 28 \\
\hline & Promedio & $\begin{array}{l}\text { Desviación } \\
\text { Estándar }\end{array}$ & Promedio & $\begin{array}{l}\text { Desviación } \\
\text { Estándar }\end{array}$ & Promedio & $\begin{array}{r}\text { Desviación } \\
\text { Estándar }\end{array}$ & Promedio & $\begin{array}{l}\text { Desviación } \\
\text { Estándar }\end{array}$ & Promedio & $\begin{array}{r}\text { Desviación } \\
\text { Estándar }\end{array}$ \\
\hline $\mathrm{SiO}_{2}$ & 93.65 & 3.84 & 93.22 & 1.71 & 92.10 & 1.29 & 91.40 & 0.92 & 94.22 & 0.34 \\
\hline $\mathrm{Al}_{3} \mathrm{O}_{3}$ & 0.28 & 0.13 & 0.31 & 0.20 & 0.25 & 0.12 & 0.57 & 0.03 & 0.36 & 0.04 \\
\hline $\mathrm{Fe}_{2} \mathrm{O}_{3}$ & 0.58 & 2.26 & 1.12 & 0.86 & 0.79 & 0.70 & 3.86 & 0.41 & 0.10 & 0.01 \\
\hline $\mathrm{CaO}$ & 0.27 & 0.07 & 0.44 & 0.34 & 0.38 & 0.11 & 0.73 & 0.08 & 0.27 & 0.05 \\
\hline $\mathrm{MgO}$ & 0.25 & 0.26 & 1.08 & 0.29 & 0.35 & 0.10 & 0.44 & 0.05 & 0.20 & 0.02 \\
\hline $\mathrm{Na}_{2} \mathrm{O}$ & 0.02 & 0.02 & 0.10 & 0.06 & 0.17 & 0.04 & 0.20 & 0.02 & - & - \\
\hline $\mathrm{K}_{2} \mathrm{O}$ & 0.49 & 0.24 & 1.37 & 0.45 & 0.96 & 0.22 & 1.06 & 0.05 & - & - \\
\hline S & 0.20 & 0.16 & 0.22 & 0.06 & - & - & - & - & - & - \\
\hline $\mathrm{SO}_{3}$ & - & - & - & - & 0.36 & 0.10 & 0.36 & 0.16 & - & - \\
\hline C & 3.62 & 0.96 & 1.92 & 1.15 & - & - & - & - & 3.05 & 0.25 \\
\hline Pérdida al Fuego & 4.36 & 1.48 & 3.10 & 0.90 & 3.20 & 0.45 & 2.62 & 0.42 & 3.60 & 0.33 \\
\hline
\end{tabular}


TABLA 3. VARIACIONES EN PROPIEDADES FÍSICAS DE MICROSÍLICE RECOPILADAS DE DISTINTAS FUENTES POR ACI-211

\begin{tabular}{|c|c|c|c|c|c|}
\hline \multicolumn{2}{|l|}{ TIPO DE ALEACIÓN } & sI & FESI-75\% & SI Y FESI- $75 \%$ & FESI-75\% \\
\hline \multicolumn{2}{|l|}{ NÚMERO DE MUESTRAS } & 24 & 24 & 32 & 30 \\
\hline \multirow{2}{*}{ Porcentaje retenido en Tamiz №. $325(45 \mu \mathrm{m})$} & Promedio & 5.40 & 1.80 & 5.62 & 3.73 \\
\hline & Desviación Estándar & 4.00 & 1.50 & 1.69 & 4.48 \\
\hline \multirow{2}{*}{$\begin{array}{l}\text { Área superficial medida utilizando el método de } \\
\text { absorción de nitrógeno }(\mathrm{m} 2 / \mathrm{Kg})\end{array}$} & Promedio & 20000 & 17200 & - & - \\
\hline & Desviación Estándar & 2100 & - & - & - \\
\hline \multirow{2}{*}{ Gravedad Específica } & Promedio & - & - & 2.27 & 2.26 \\
\hline & Desviación Estándar & - & - & 0.02 & 0.08 \\
\hline \multirow{2}{*}{$\begin{array}{l}\text { Índice de actividad puzolánico con Cemento Portland } \\
(\%)\end{array}$} & Promedio & 102.80 & 96.50 & 91.90 & 95.30 \\
\hline & Desviación Estándar & 5.10 & 13.70 & 10.00 & 4.00 \\
\hline \multirow{2}{*}{ índice de actividad puzolánico cal (MPa) } & Promedio & 8.90 & - & 7.00 & 9.10 \\
\hline & Desviación Estándar & 0.80 & - & 0.80 & 0.90 \\
\hline \multirow{2}{*}{ Demanda de Agua (\%) } & Promedio & 138.80 & 139.20 & 140.10 & 144.40 \\
\hline & Desviación Estándar & 4.20 & 7.20 & 2.60 & 2.00 \\
\hline
\end{tabular}

Se utilizaron dos aditivos distintos, ambos acorde a la norma ASTM 494 para aditivos reductores de agua de alto rango. El primero (ADTV1), denominado de última generación, que trabaja con policarboxilato como agente activo; estos aditivos son reconocidos hasta la fecha como los más apropiados para el desarrollo de hormigones de alto desempeño [2]. El segundo (ADTV2) es un aditivo de segunda generación, utilizado para la producción de concreto reoplástico, utilizado por motivos de comparación. Ambos aditivos se encuentran en estado diluido, desconociéndose su concentración exacta, por lo que siguiendo el método de Willie et. al. [2], se asumió un $40 \%$ de contenido sólido con el fin de tomar en cuenta la incorporación de agua adicional a la mezcla.

AGREGADOS GRUESOS

El desarrollo de hormigones de alta resistencia requiere una selección cuidadosa de agregados, debido a que éstos son los que ocuparán mayor volumen en la mezcla final y tendrán un impacto significativo en las propiedades del hormigón [1]. En hormigones de alto desempeño, aun cuando la matriz del cementante tenga buenas cualidades, es posible que la máxima resistencia del hormigón esté limitada por la resistencia del agregado. Por ello, se recomienda utilizar agregados resistentes, limpios, sin fisuras y tomar en cuenta que su forma, tamaño, granulometría y composición mineralógica afectarán las características finales del hormigón y la demanda de agua de la mezcla [1].

Para las mezclas desarrolladas se utilizaron varios tipos de agregados distintos, terminándose por seleccionar los agregados provenientes de procesos de trituración y clasificación del material pétreo del Río Pilatón, cercano a uno de los proyectos hidroeléctricos para los cuales se diseña la mezcla de hormigón de alto desempeño. El proceso permite obtener agregados de superficie angular y con baja presencia de partículas alargadas y planas. La granulometría de estos agregados, de tamaño nominal máximo 3/4 (AGR1) y de tamaño nominal máximo 3/8 (AGR2) se detallan en las figuras 1 y 2.

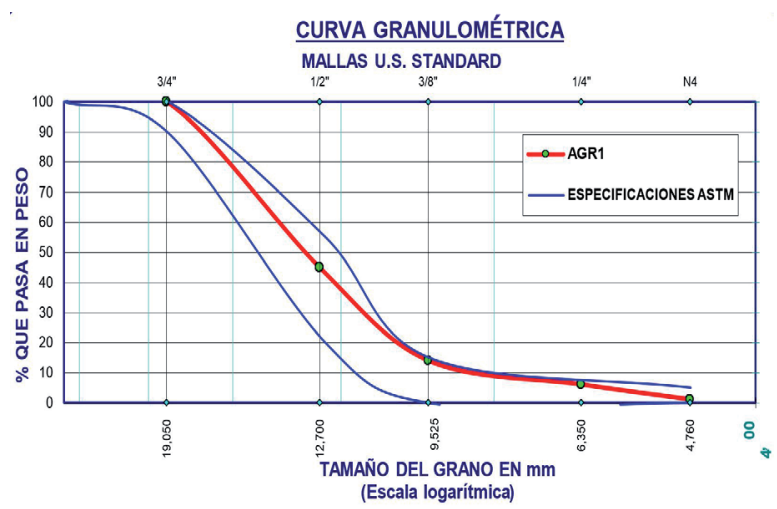

Figura 1. Granulometría del agregado grueso AGR1 (3/4").

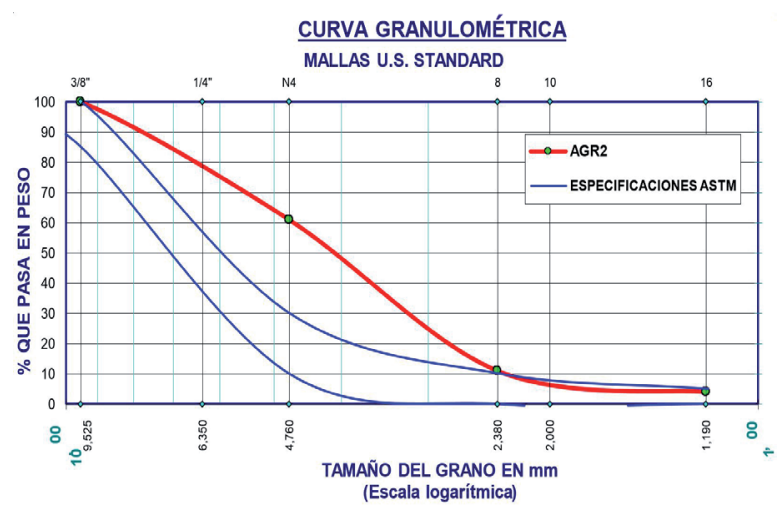

Figura 2. Granulometría del agregado grueso AGR2 (3/8"). 
AGREGADOS FINOS

las características del agregado fino utilizado pueden tener efectos de similar importancia a las del agregado grueso, por lo que se utilizan los requerimientos ASTM C33 y se recomienda un módulo de finura (MF) entre 2.5 y 3.2, o incluso mayor, con el fin de evitar problemas de trabajabilidad y un aumento excesivo de la demanda de agua [1]. En el presente trabajo se utilizaron agregados finos (AFR1 y AFR2) provenientes de la misma fuente de producción del agregado grueso, cuyas granulometrías se muestran en las figuras 3 y 4.

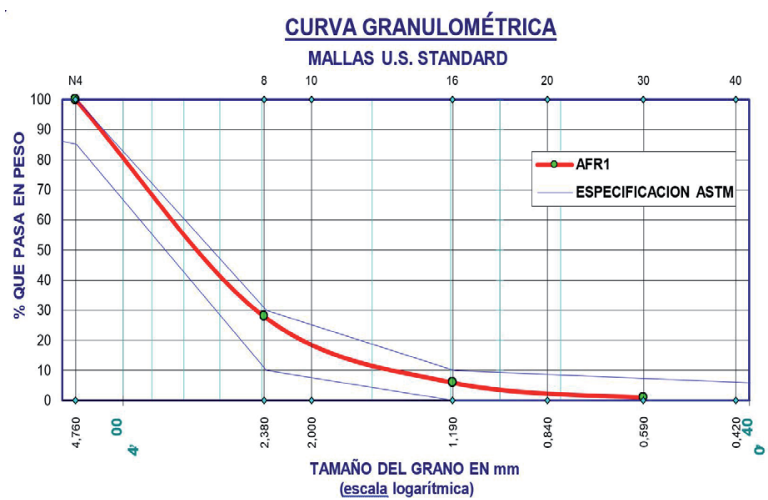

Figura 3. Granulometría del agregado fino (arena gruesa) AFR1 (\#4).

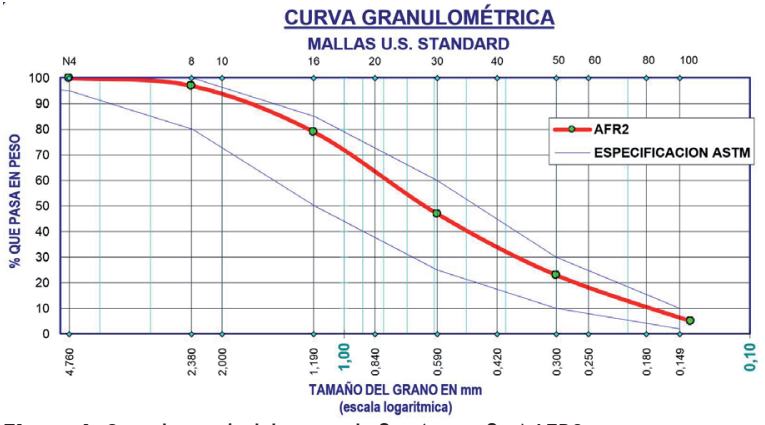

Figura 4. Granulometría del agregado fino (arena fina) AFR2.

AGUA

se utilizó agua proveniente del sistema de suministro de la ciudad de Quito, práctica aceptable por la buena calidad de la misma. De sospechar algún problema debido a la calidad del agua utilizada, ACI-211 recomienda referirse a la norma ASTM C1603 para agua utilizada en mezclas de hormigón [1].

REQUERIMIENTOS

Esta investigación se concentra en tres requerimientos de desempeño: alta resistencia a la compresión, trabajabilidad de mezcla y resistencia a la erosión abrasión. El primer requerimiento es obtener resistencias a la compresión de alrededor de $90 \mathrm{MPa}$, evaluadas mediante ensayos de resistencia a la compresión acorde a ASTM C31 y C39. El segundo requerimiento considerado fue la obtención de una trabajabilidad adecuada en la etapa fresca del hormigón evaluada mediante ensayos de revenimiento acorde a ASTM C143, con un mínimo de 5". No se estableció un asentamiento máximo puesto que, aunque un asentamiento alto puede ser un indicador de posible segregación [5], la adición de microsílice hace al hormigón resistente a este fenómeno [3, 4] y niveles altos de asentamiento pueden deberse a efectos de los aditivos reductores de agua. Finalmente, se requiere un hormigón que presente bajos índices de desgaste a la erosión - abrasión, de no más de un $2 \%$ de desgaste según norma ASTM C1138.

\section{DOSIFICACIÓN INICIAL}

Se tomaron en cuenta tres parámetros principales para la dosificación de las diferentes mezclas. La cantidad de cemento y las relaciones microsílice/cemento (SF/C) y agua/cementante $(\mathrm{w} / \mathrm{c})$. ACI recomienda mantener el contenido de microsílice entre un 5 y 15\% de la masa de cemento y relaciones w/c tan bajos como 0.20 [1]. Para obtener una dosificación inicial se analizaron aquellas propuestas por los comités ACI consultados [1, 3] y por Holland T. [4]. La predosificación 1 fue extraída del "Silica Fume User's Manual” [4] y la predosificación 2 del comité ACI211.4R-08 [1]. Ambas se muestran en la tabla 4 junto con los parámetros calculados. Ambas predosificaciones utilizan cementantes adicionales que se escapan del alcance de esta investigación (cemento de escoria y cenizas volantes) por lo que el procedimiento seguido fue reemplazar estos cementantes por un peso similar del cemento utilizado en la mezcla (CPII o CH-HE).

A partir de las pruebas iniciales con las predosificaciones, se escogieron cinco dosificaciones volumétricas con los dos cementos estudiados, las cuales se detallan en la tabla 5. Las mezclas 1 y 2 se diseñaron acorde a la predosificación 1 variando únicamente el tipo de cemento. Las mezclas 3, 4 y 5 siguen los lineamientos de la predosificación dos; entre las mezclas 3 y 4 la diferencia se establece en el procedimiento seguido para la preparación de las muestras, como se describe a continuación.

\section{PREPARACIÓN DE MUESTRAS}

Para cada una de las dosificaciones propuestas se fabricaron muestras a escala, siguiendo dos procedimientos distintos, uno para las muestras 1-3 y otro para las muestras 4 y 5 . 
La preparación de muestras incluye las correcciones necesarias en la cantidad de agua de mezcla, con la intención de obtener una trabajabilidad adecuada y una mezcla homogénea de los componentes del hormigón. Para todas las mezclas se utilizó una mezcladora de $1 / 3$ de saco que fue previamente limpiada y llevada a un estado de saturación de su superficie de modo que no extraiga o aporte agua a la mezcla. Para las mezclas 1-3 se inició por verter el agua en la mezcladora, seguida del agregado grueso. Posteriormente se añadieron los cementantes y el aditivo, seguidos por los agregados finos. Se mezcló durante cuatro minutos seguidos de cuatro minutos de reposo y cuatro minutos de mezclado finales. Durante el reposo se evaluó la apariencia física de la mezcla buscando que ésta sea fluida y que los cementantes hayan formado una pasta que recubra a los agregados. Durante los últimos
4 minutos de mezclado se añadió agua en pequeñas cantidades de ser necesario con la intención de que el hormigón llegue a tener al aspecto deseado.

Para las mezclas 4 y 5 se inició de igual manera saturando la superficie de la mezcladora y colocando el agregado grueso. Posteriormente los cementantes junto con el agua y el aditivo fueron premezclados aparte utilizando una batidora; la pasta formada fue luego añadida a la mezcladora seguida de los agregados finos. Este procedimiento pareció ser más efectivo en facilitar la reacción de los cementantes con el aditivo y permitir la obtención de una mezcla fluida. La mezcladora se mantuvo en funcionamiento durante cuatro minutos, seguidos de cuatro minutos de descanso y cuatro minutos finales de mezclado durante los cuales se añadió agua de ser necesario, al igual que en las mezclas 1-3.

\section{TABLA 4. PREDOSIFICACIONES INICIALES ADOPTADAS}

\begin{tabular}{lll}
\hline No. & $\mathbf{1}$ & $\mathbf{2}$ \\
\hline Fuente & Silica Fume User's Manual & ACl211.4R-08 \\
\hline Resistencia 28 días $[\mathrm{MPa}]$ & 83 & 107 \\
\hline Cemento Portland $[\mathrm{Kg} / \mathrm{m} 3]$ & 406 & 473.68 \\
\hline Ceniza Volante $[\mathrm{Kg} / \mathrm{m} 3]$ & 0 & 103.62 \\
\hline Cemento de Escoria $[\mathrm{Kg} / \mathrm{m} 3]$ & 169 & 0 \\
\hline Microsílice (SF) $[\mathrm{Kg} / \mathrm{m} 3]$ & 47 & 74.01 \\
\hline Tot. Cementantes (C) $[\mathrm{Kg} / \mathrm{m} 3]$ & 622 & 651.32 \\
\hline SF/C & $7.56 \%$ & $11.36 \%$ \\
\hline W/C & 0.2 & 0.2 \\
\hline
\end{tabular}

TABLA 5. DOSIFICACIONES INICIALES PARA PRODUCIR 1 M3 DE HORMIGÓN

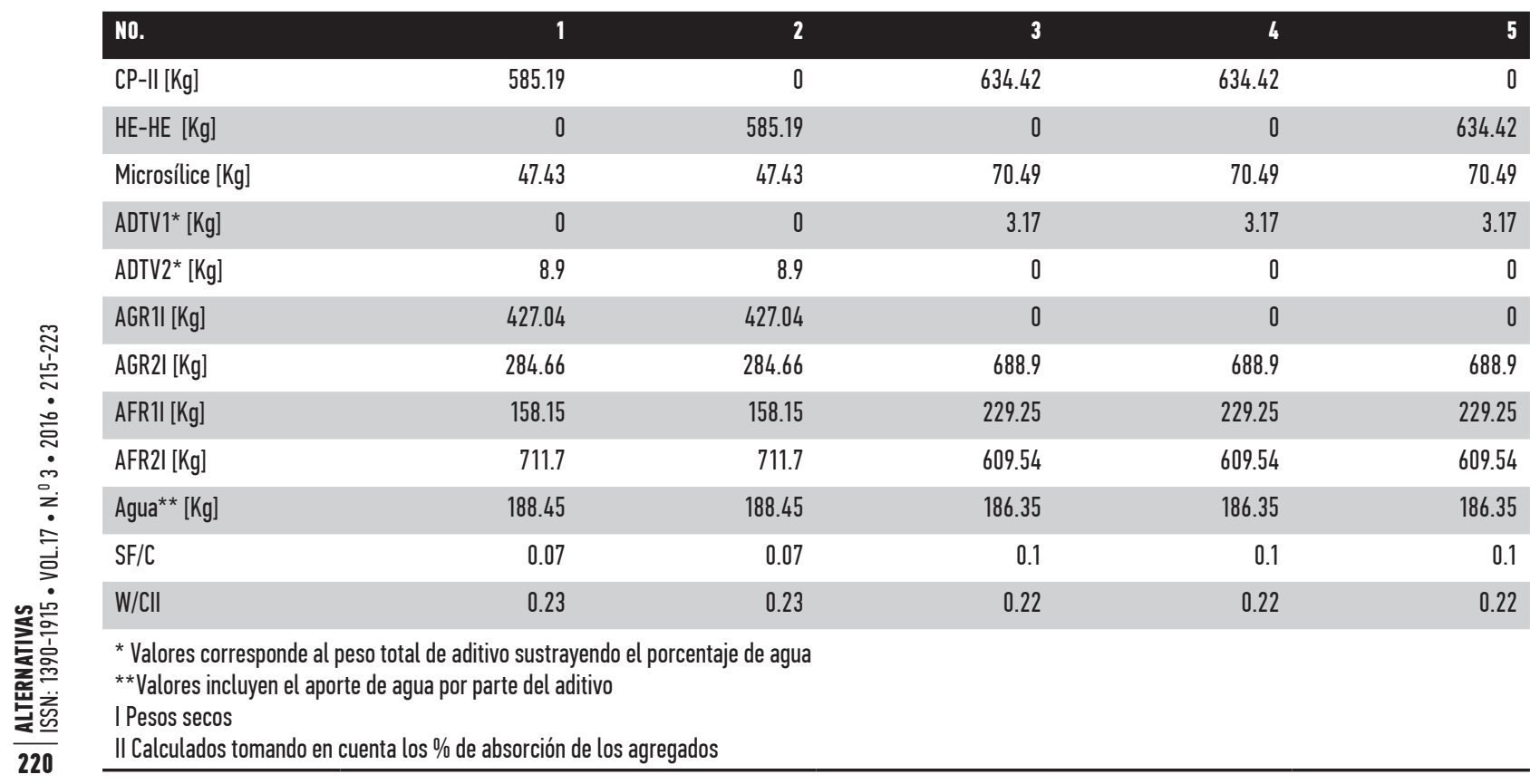


TABLA 6. DOSIFICACIONES FINALES PARA PRODUCIR 1 M3 DE HORMIGÓN

\begin{tabular}{|c|c|c|c|c|c|c|}
\hline NO. & [KG] & 1 & 2 & 3 & 4 & 5 \\
\hline CP-II [Kg] & Agua** $[\mathrm{Kg}]$ & 585.19 & 0 & 634.42 & 634.42 & 0 \\
\hline HE-HE [Kg] & $S F / C$ & 0 & 585.19 & 0 & 0 & 634.42 \\
\hline Microsílice [Kg] & W/CII & 47.43 & 47.43 & 70.49 & 70.49 & 70.49 \\
\hline $\mathrm{ADTV}^{\star}[\mathrm{Kg}]$ & & 0 & 0 & 3.17 & 3.17 & 3.17 \\
\hline ADTV2* [Kg] & & 8.9 & 8.9 & 0 & 0 & 0 \\
\hline AGR1I [Kg] & & 427.04 & 427.04 & 0 & 0 & 0 \\
\hline AGR2I [Kg] & & 284.66 & 284.66 & 688.9 & 688.9 & 688.9 \\
\hline AFR1l [Kg] & & 158.15 & 158.15 & 229.25 & 229.25 & 229.25 \\
\hline AFR21 [Kg] & & 711.7 & 711.7 & 609.54 & 609.54 & 609.54 \\
\hline \multirow[t]{4}{*}{ Agua añadida } & & 5.49 & 30.95 & 3.02 & -10.07 & 25.18 \\
\hline & & 193.94 & 219.4 & 189.37 & 176.28 & 211.52 \\
\hline & & 0.07 & 0.07 & 0.1 & 0.1 & 0.1 \\
\hline & & $24 \%$ & $28 \%$ & $23 \%$ & $21 \%$ & $26 \%$ \\
\hline \multicolumn{7}{|c|}{$\begin{array}{l}\text { *Valores corresponde al peso total de aditivo sustrayendo el porcentaje de agua } \\
\text { **Valores incluyen el aporte de agua por parte del aditivo }\end{array}$} \\
\hline
\end{tabular}

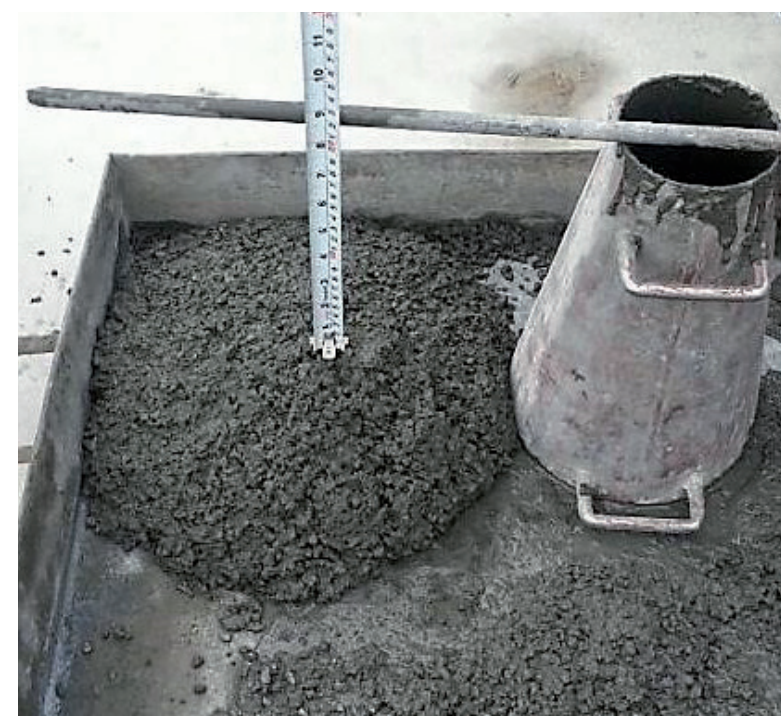

Figura 5. Revenimiento típico en muestras de la mezcla 4.

RESULTADOS

La variación en estos procedimientos es necesaria, conforme se gana experiencia en la manera cómo interactúan el cemento, la microsílice y el aditivo reductor de alto rango. Las variaciones en el agua de mezcla han sido tomadas en cuenta en la tabla 6 que muestra las dosificaciones finales para cada una de las cinco mezclas realizadas.

Finalizados los tiempos de mezclado de cada una de las dosificaciones, se procedió a realizar pruebas de revenimiento, acorde con la norma ASTM C143, en la cual todas las muestras mostraron asentamientos mayores a las cinco pulgadas fijadas como requerimiento mínimo (figura 5). Finalmente, se procedió a la fabricación de mues- tras cilíndricas y a su posterior curado acorde con la norma ASTM C31. Las pruebas a compresión se realizaron a los 7 y 28 días siguiendo las recomendaciones de la norma ASTM C39.

\section{RESISTENCIA A LA COMPRESIÓN Y REVENIMIENTO}

Las resistencias obtenidas en las pruebas a compresión a los 28 días se consideran resistencias características Fcr, conforme lo especifica el comité ACI-211 [1]. Siguiendo la misma especificación, la resistencia a la compresión a los 28 días f'c, definida como requisito de aceptación del hormigón, es menor, basada en las variaciones estadísticas de los resultados de los ensayos. Los resultados de la resistencia f'c se muestran en la tabla 7. Como puede observarse, la mezcla 4 correspondiente al cemento portland tipo II dio como resultado una resistencia última a la compresión f'c de 109.88 MPa, equivalente a una resistencia Fcr del orden de 126.51 MPa. Algunas probetas de dicha mezcla alcanzaron incluso valores extremos de $138 \mathrm{MPa}$ $\left(1407 \mathrm{kgf} / \mathrm{cm}^{2}\right)$, siendo este el hormigón de mayor resistencia producido en Ecuador del que se tenga algún registro hasta la fecha, con una gran trabajabilidad, medida en 9" de revenimiento en promedio.

RESISTENCIA A LA EROSIÓN - ABRASIÓN

Para la evaluación de la resistencia a la erosión - abrasión de las mezclas preparadas, se realizaron ensayos según norma ASTM C1138, la cual evalúa porcentajes de pérdida de masa de probetas cilíndricas de aproximadamente 30 
$\mathrm{cm}$ de diámetro y $10 \mathrm{~cm}$ de espesor, sometidas a la abrasión por rotación de bolas de acero en la superficie de la probeta, a velocidades de rotación de $1260 \mathrm{rpm}$, y a diferentes tiempos de ensayo. Los resultados de los desgastes de las muestras se indican en la figura 6 , mostrando un desgaste máximo $0.79 \%$ a las 72 horas, valor mucho menor que el de diseño, de $2 \%$. El equipo utilizado junto con la característica del hormigón sometido a erosión abrasión puede observarse en las figuras 7 y 8 .

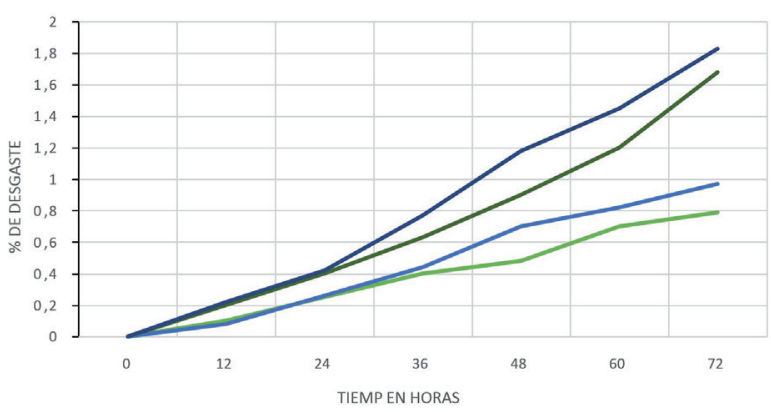

Figura 6. Resultados de pruebas de desgaste según ensayo ASTM C1138.

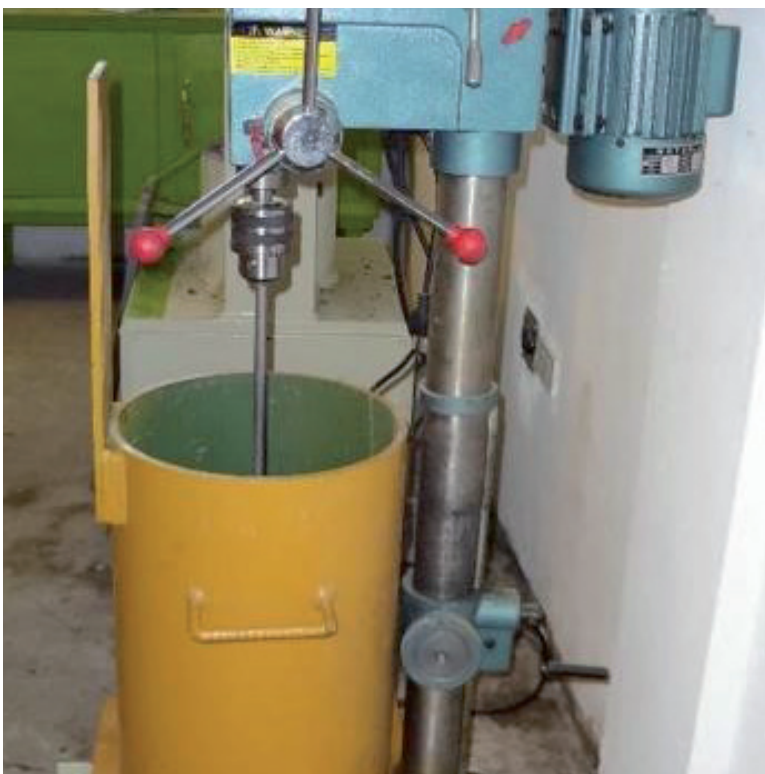

Figura 7. Equipo utilizado para pruebas de erosión abrasión.

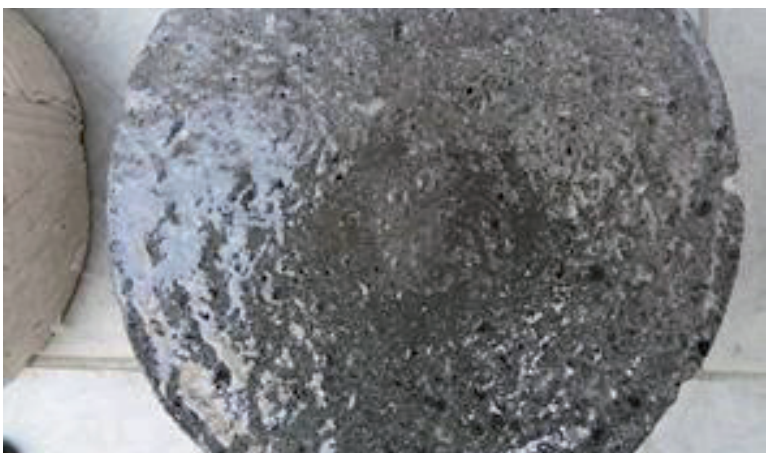

Figura 8. Probeta de mezcla 4 luego del ensayo de erosión abrasión.
TABLA 7. RESISTENCIA FINAL A LA COMPRESIÓN F'C DE LAS MEZCLAS FINALES

\begin{tabular}{rr} 
MEZCLA NO. & RESISTENCIA A LA COMPRESIÓN F'C [MPA] \\
\hline 1 & 70.33 \\
2 & 76.8 \\
3 & 98.9 \\
4 & 109.92 \\
\hline 5 & 77.91 \\
\hline
\end{tabular}

TABLA 8. PRECIOS UNITARIOS

\begin{tabular}{lrlr}
\hline MATERIAL & PRECIO [USS] & UNIDAD & PRECIO POR 1 KG [USS] \\
\hline Cemento & 7.8 & Saco $50 \mathrm{Kg}$ & 0.156 \\
\hline Microsílice & 900 & Ton & 0.9 \\
\hline ADTV1 & $5.3 \mathrm{~kg}$ & 5.3 \\
ADTV2 & $2.45 \mathrm{Kg}$ & 2.45 \\
\hline Agregados & $36 \mathrm{~m} 3$ & 0.013 \\
\hline
\end{tabular}

TABLA 9. RELACIÓN RESISTENCIA/COSTO

\begin{tabular}{|lrrr|}
$\begin{array}{l}\text { MEZCLA } \\
\text { NO. }\end{array}$ & $\begin{array}{r}\text { RESISTENCIA A LA } \\
\text { COMPRESIÓN F'C IMPA] }\end{array}$ & $\begin{array}{r}\text { PRECIO POR } \\
\text { M }^{3} \text { [USS] }\end{array}$ & $\begin{array}{r}\text { RELACIÓN } \\
\text { RESISTENCIA/COSTO }\end{array}$ \\
\hline 1 & 70.33 & 227.55 & 0.31 \\
\hline 2 & 76.8 & 227.89 & 0.34 \\
\hline 3 & 98.9 & 238.64 & 0.41 \\
\hline 4 & 109.92 & 238.46 & 0.46 \\
\hline 5 & 77.91 & 238.93 & 0.33 \\
\hline
\end{tabular}

RELACIÓN RESISTENCIA/COSTO

Los costos de producción de las mezclas se obtuvieron en base a un análisis de precios unitarios, utilizando datos del mercado ecuatoriano. Los costos indicados incluyen el transporte de los materiales y se muestran en la tabla 8. Los costos por metro cúbico de producción y las relaciones resistencia/costo de cada una de las mezclas se indican en la tabla 9, observándose nuevamente a la mezcla No. 4 con la mayor relación resistencia costo. De manera interesante, el costo de la mejor mezcla resultó ser también la más económica y la más eficiente de todas.

RESULTADOS

En general el procedimiento seguido para obtener las predosificaciones ha demostrado ser efectivo. El comparar las resistencias esperadas de la tabla 4 con las resistencias finales de la tabla 7, parece indicar que el sustituir cementantes adicionales propuestos por las predosificaciones 1 y 2 (cemento de escoria y ceniza volante) por los cementos $\mathrm{CH}-\mathrm{HE}$ y CPII, acarrea una disminución de la resistencia final esperada pero no impide el desarrollo de hormigones de alta resistencia. Sin embargo, la mezcla 4 ha logrado superar la resistencia 
esperada por la predosificación 2, sin la incorporación de ceniza volante (probablemente debido al método de mezclado más efectivo y una actitud conservadora de las fuentes consultadas y descritas en la tabla 4 , al especificar las resistencias esperadas). Los resultados muestran que el cemento $\mathrm{CH}-\mathrm{HE}$ alcanzó una resistencia mayor al cemento CP-II al utilizar como base la predosificación 1 . Sin embargo al utilizar los parámetros de la predosificación 2 el cemento CP-II es el más apropiado.

Observando los datos de corrección de agua se puede notar, que el cemento $\mathrm{CH}$-HE produce consistentemente una demanda de agua mayor al cemento CP-II y por tanto dificulta la consecución de relaciones w/c bajas. El procedimiento utilizado para mezclar los materiales también demostró tener un impacto considerable en la demanda de agua. Comparando las mezclas 3 y 4 puede observarse, que el cambio total en el agua requerida fue de $260 \mathrm{~kg}$ y llevó a un cambio en la relación w/c de 0.23 a 0.21 , factor que explica un aumento en la resistencia final de casi $11 \mathrm{MPa}$.

Todos los tipos de agregados utilizados demostraron ser aptos para el desarrollo de hormigones de alta resistencia. La efectividad de su granulometría y el buen empaquetamiento obtenido se puede observar en la figura 9 que muestra la superficie de fallo de uno de los cilindros de prueba. El hormigón de alta resistencia resultó también tener excelente resistencia a la erosión abrasión con una adecuada trabajabilidad para su producción, lográndose los objetivos de desempeño buscados.

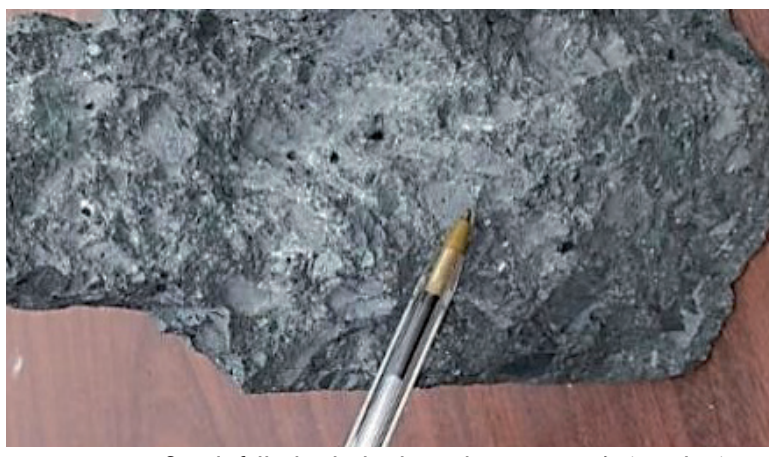

Figura 9. Superficie de falla de cilindro de prueba a compresión (mezcla 4).
Acerca de los aditivos utilizados, los resultados indican que el reductor de agua de última generación en base a policarboxilatos fue el más eficaz, lográndose relaciones w/c más bajas para cada tipo de cemento, que las alcanzadas con el aditivo de segunda generación.

Para los fines perseguidos en esta investigación, se considera que los datos obtenidos son suficientes. Sin embargo, para una producción industrial es necesario obtener la resistencia característica y su relación con f'c con base a las desviaciones estándar de producción. Es fundamental mantener un control de calidad muy exigente en la producción de este tipo de hormigones, pues pequeñas variaciones en las propiedades de los materiales utilizados generan variaciones en los resultados del producto final y requiere de ajustes de dosificación rutinarios.

\section{AGRADECIMIENTOS}

Los autores dejan constancia de su agradecimientos a la empresa Proyectos y Construcciones Hidroenergéticas PCH Cía. Ltda. por facilitar el acceso a su laboratorio de materiales y hormigones y a su personal técnico.

\section{REFERENCIAS BIBLIOGRÁFICAS}

1. ACI 211.4R-08. 2008. "Guide for Selecting Proportions for High-Strength Concrete Using Portland Cement and Other Cementitious Materials", American Concrete Institute, 29p.

2. Willie, K.; Naamany, A; Parra-Montesinos, G. 2011. "Ultra-High Performance Concrete with Compressive Strength Exceeding $150 \mathrm{MPa}$ (22ksi): A Simpler Way". ACI Materials Journal, 108(M06), 46-54.

3. ACI 234R-06. 2006. "Guide for the Use of Silica Fume in Concrete", American Concrete Institute, 63p.

4. Holland, T. 2005. "Silica Fume User's Manual," Silica Fume Association, Lovettsville, VA, Rep. FHWA-IF-05-016, 1-77.

5. Philips, W. et. al. 2005. "Capítulo 2: La mezcla del concreto," Guía del Contratista Para la Construcción en Concreto de Calidad, 3ra ed. USA, ACI \& ASCC, 2: 14-27. 